\title{
Rabbit Model of Lyme Borreliosis: Erythema Migrans, Infection-derived Immunity, and Identification of Borrelia burgdorferi Proteins Associated with Virulence and Protective Immunity
}

\author{
Denise M. Foley, ${ }^{*}$ Richard J. Gayek, ${ }^{\ddagger}$ Jon T. Skare, ${ }^{*}$ Elizabeth A. Wagar, ${ }^{5}$ Cheryl I. Champion, ${ }^{*}$ David R. Blanco," \\ Michael A. Lovett, *\|l and James N. Miller* \\ "Department of Microbiology and Immunology, ${ }^{\ddagger}$ Division of Laboratory Animal Medicine, ${ }^{8}$ Department of Pathology, and "Division of \\ Infectious Diseases, Department of Medicine, University of California, Los Angeles, School of Medicine, Los Angeles, California 90024
}

\begin{abstract}
Erythema migrans (EM), persistent skin infection, and visceral dissemination can be induced reproducibly in the adult male New Zealand White rabbit by intradermal injection of as few as $10^{3}$ Borrelia burgdorferi. EM was found to persist for $7 \pm 3 \mathrm{~d}$. Skin culture positivity (infection) cleared within a mean of $6.7 \pm 1.4$ wk after infection and similarly visceral infection was not demonstrated after 8 wk; infection-derived immunity to intradermal challenge was evident 5 mo after initial infection. The extent of the protection against EM and dermal infection induced by untreated infection was directly related to the extent of prior in vitro passage of the B31 strain. Initial infection with as few as 4 $\times 10^{3}$ B31 passage 4 induced complete protection against EM and skin infection upon subsequent challenge with 4 $\times 10^{7} \mathrm{B31}$, passage 4. Initial infection with $\mathrm{B31}$ passage 27 led to partial protection against EM along with complete protection against skin infection. Initial infection with passage 47 led to partial protection against EM, but conferred no protection against skin infection. Using serum from rabbits fully immune to reinfection, we defined a set of $B$. burgdorferi proteins present in virulent B31, but absent in the avirulent American Type Culture Collection B31 strain, termed "va" for virulent strain associated. The va proteins of $B 31$ passages 1, 27, and 47 differed strikingly, thus raising the possibility that these changes may relate in a causal way to the differences in induction of protective immunity observed. (J. Clin. Invest. 1995. 96:965-975.) Key words: Borrelia burgdorferi - Lyme borreliosis - Lyme disease • erythema migrans $\cdot$ rabbit model
\end{abstract}

\section{Introduction}

Lyme borreliosis, the most common arthropod vector-borne disease in the United States (1), is caused by the spirochete

This work was presented in part at the Cold Spring Harbor Symposium, 30 March 1993 and has appeared in abstract form (94th American Society of Microbiology General Meeting 23-27 May 1994. Las Vegas, NV. Abstract \#D165:125.)

Address correspondence to James N. Miller, Department of Microbiology and Immunology, UCLA School of Medicine, 10833 Le Conte Avenue, Los Angeles, CA 90024-1747. Phone: 310-825-1979; FAX: 310-206-3865.

Received for publication 29 September 1994 and accepted in revised form 25 April 1995.

J. Clin. Invest.

(c) The American Society for Clinical Investigation, Inc.

$0021-9738 / 95 / 08 / 0965 / 11 \quad \$ 2.00$

Volume 96, August 1995, 965-975
Borrelia burgdorferi sensu lato. In the majority of patients, an early manifestation of infection is the skin lesion, erythema migrans $(E M)^{1}(2,3)$. These large, distinctive macular skin lesions may be uniformly erythematous, or "target" or "banded"' in appearance. Histopathologically, EM is marked by perivascular infiltration of lymphocytes, macrophages, and plasma cells (4). Early Lyme disease may also be characterized by spirochetal dissemination with subsequent involvement of joints, central and peripheral nervous systems, lymphoid tissue, and the heart. $(2,5-8)$.

There are a variety of rodent models, including rats $(9,10)$, mice $(11,12)$, hamsters $(13-15)$, gerbils $(16)$, and guinea pigs (17) where $B$. burgdorferi can establish disseminated and chronic infection $(10,12,15-17)$. However, only in the rhesus monkey model has EM been reported (18). Previous attempts to produce EM in the rabbit have not resulted in lesion formation from which $B$. burgdorferi has been isolated or which exhibit the characteristic histopathology of EM (19-21) However, polyarthritis and carditis, as well as dissemination of $B$. burgdorferi to spleen, kidney, brain, and joint have been reported for 3-wk-old American Dutch rabbits (22).

In this study, using adult male New Zealand White rabbits, we report the reproducible induction of lesions with the appearance and histopathologic features of EM. B. burgdorferi sensu stricto persists in the skin and disseminates to popliteal lymph nodes, stifle (knee) joints, and the spinal cord. Within a mean of $7 \mathrm{wk}$, infection is cleared from all cutaneous sites. Clearance of visceral infection was found to correlate closely with the clearance of dermal infection; no evidence of visceral infection was found after $8 \mathrm{wk}$. After clearance, rabbits exhibit immunity to intradermal (ID) challenge with virulent $B$. burgdorferi. Study of this infection-derived immunity enabled us to identify B. burgdorferi molecules of potential relevance to virulence and to induction of protective immunity in the rabbit. The rabbit model provides unique opportunities to study both the early events in the pathogenesis of Lyme borreliosis and the nature of infection-derived immunity.

\section{Methods}

Borrelia burgdorferi strains. Strains used and their respective sources are shown in Table I. Strains were grown at $34^{\circ} \mathrm{C}$ in $5 \mathrm{ml}$ of BSK II medium (23). When the cultures reached maximum density, $1.0 \mathrm{ml}$ was passaged (subcultured) into $5 \mathrm{ml}$ of fresh medium. Initial passage from tissue was designated "passage 0 " and subsequent passages desig-

1. Abbreviations used in this paper: ATCC, American Type Culture Collection; EM, erythema migrans; ID, intradermal; PVDF, polyvinylidene diflouride; va, virulent strain associated. 
Table I. B. burgdorferi Strains Used in this Study and Their Sources

\begin{tabular}{llll}
\hline $\begin{array}{c}\text { B. burgdorferi } \\
\text { strain }\end{array}$ & \multicolumn{1}{c}{ Isolate } & & Source \\
\hline B31 & Ixodes scapularis Shelter Island & Stephen Barthold, Yale University, New Haven, CT \\
N40 & Ixodes scapularis Shelter Island & & 20, 23 \\
HB19 & Human blood patient in CT & & " \\
SH-2-82 & Ixodes scapularis Shelter Island & Thomas Schwan, Rocky Mountain Research Laboratories. NIAID. Hamilton, MT & 25 \\
CA-2-87 & Ixodes pacificus California & & 25 \\
ECM-NY-86 & Human skin patient in NY & & 25 \\
297 & Ixodes scapularis Shelter Island & Russell Johnson, Univeristy of Minnesota, MN \\
B31 avirulent & Ixodes scapularis Shelter Island & ATCC cat. No. 35210 & 5 \\
& & & 20,23 \\
\hline
\end{tabular}

nated by consecutive numbers. This method of passage is similar to one previously described (24). Fully grown cultures were frozen in $10 \%$ DMSO and stored at $-80^{\circ} \mathrm{C}$. Cultures frozen at a particular passage were designated as the next passage upon thawing and cultivation. The infectivity of the B31 strain for mice is described below.

Experimental animals. $\mathrm{C} 3 \mathrm{H} / \mathrm{HeJ}$ mice 3 wk of age (Jackson Laboratories, Bar Harbor, ME) were housed in cages containing no more than five animals. Infected and noninfected mice were housed separately. Adult male New Zealand White rabbits 6-9 mo of age (Irish Farms, Norco, CA) were housed individually in a temperature controlled environment ranging from 18 to $23^{\circ} \mathrm{C}$. Before each ID inoculation of $\boldsymbol{B}$. burgdorferi, the backs of rabbits were clipped closely with an electric clipper fitted with a size 40 blade to expose the skin (Oster Professional Products, McMinnville, TN).

Preparation of B. burgdorferi strains for inoculation. Strains utilized in experiments for mouse inoculations were diluted to the indicated numbers in BSK II medium and inoculated intradermally in $0.1-\mathrm{ml}$ volumes on the abdomen of each mouse. B. burgdorferi suspensions used in the rabbit experiments were all reisolated from infected mice or rabbits. Cultures were harvested by centrifugation at $8,000 \mathrm{~g}$ and washed three times in normal rabbit serum which had been heat inactivated at $56^{\circ} \mathrm{C}$ for 30 min then diluted 1:1 in PBS, pH 7.2 (NRS-PBS). After each centrifugation and resuspension, spirochetal motility became less active; as a result, the spirochetes were allowed to sit at room temperature for $15 \mathrm{~min}$ to regain maximum motility. Organisms used as heat-killed controls were placed at $56^{\circ} \mathrm{C}$ for $45 \mathrm{~min}$ after the last wash. Concentrations of spirochetes were determined by darkfield visualization using a calibrated darkfield microscope. The inoculum was resuspended to the desired concentration after the last wash, and injected intradermally into the clipped backs of rabbits in $0.1-\mathrm{ml}$ volumes. To visualize the site of inoculation throughout the course of the experiments, injections were always administered adjacent to a black dot made with a water-resistant laboratory marker.

Mouse infectivity of B31 strains. The B31 strain was shown to be infectious for 3-wk-old $\mathrm{C} 3 \mathrm{H} / \mathrm{HeJ}$ mice after 4, 8, 27, 47, and 85 passages in BSK II medium. Each of five mice per passage exhibited positive blood, ear, spleen, and/or bladder cultures obtained after intradermal inoculation of $10^{3}-10^{7}$ spirochetes by the methods described below. The infectivity of the 27,47 , and 85 passaged spirochetes is in contrast to the findings of other investigators $(14,24)$. The American Type Culture Collection (ATCC) B31 strain, which has been passaged extensively in BSK II medium, was never isolated from inoculated $\mathrm{C} 3 \mathrm{H} / \mathrm{HeJ}$ mice (ATCC, Rockville, MD).

Punch biopsy procedure. Rabbits were anesthetized by intramuscular injection with $45 \mathrm{mg}$ Ketaset (Fort Dodge Laboratories, Fort Dodge, IA) and $8.8 \mathrm{mg}$ Xylazine (Loyd Laboratories, Shenandoah, IA) per kilogram body weight. A 4-5 mm sterile punch biopsy (Baker and Cummings, Lakewood, NJ.) was taken from the clipped back or pinnae (ear) of the rabbit. Each biopsy specimen was placed on ice in a sterile Petri dish and divided into two samples. One sample was minced and cultured in $5 \mathrm{ml}$ of BSK II medium containing $100 \mu \mathrm{g} / \mathrm{ml}$ phosphomycin and $50 \mu \mathrm{g} / \mathrm{ml}$ rifampin (25), while the other was placed in $10 \%$ buffered formalin for histopathological analysis.

Dissemination studies. $\mathrm{C} 3 \mathrm{H} / \mathrm{HeJ}$ mice $3 \mathrm{wk}$ of age were inoculated intradermally with $10^{3}-10^{7}$ actively motile spirochetes. At periods of time after inoculation, mice were killed by ether inhalation and the blood, bladder, spleen, and a portion of the pinnae removed and placed on ice until processed. Rabbits were similarly inoculated intradermally on the clipped back with $10^{3}$ or $10^{7} \mathrm{~B}$. burgdorferi per inoculated site and killed at specified time points. Before killing the rabbits, blood for culture was obtained from the central ear artery. The animals were then anesthetized as described above and cerebrospinal fluid obtained from the cisterna magna. The rabbits were killed by lethal intravenous injection of $100 \mathrm{mg} / \mathrm{kg}$ sodium pentobarbital and organs and tissues aseptically removed and placed on ice in a sterile Petri dish. Synovial fluid was obtained from the femoro-patellar capsule with a sterile swab and placed directly into BSK II. Joint tissue which surrounded the patella was taken for culture.

Isolation of B. burgdorferi strains from infected tissues. $100 \mu \mathrm{l}$ of blood or spinal fluid was inoculated into $5 \mathrm{ml}$ of BSK II medium containing $100 \mu \mathrm{g} / \mathrm{ml}$ phosphomycin and $50 \mu \mathrm{g} / \mathrm{ml}$ rifampin. Representative portions of each tissue or organ were divided into $\sim 5$-mm sections, minced, and then placed into $5 \mathrm{ml}$ of antimicrobial-containing BSK II. Cultures were incubated at $34^{\circ} \mathrm{C}$ for a period of $30 \mathrm{~d}$ and the presence or absence of $B$. burgdorferi was determined by darkfield microscopy. Cultures were considered negative when no spirochetes were observed during a $30-\mathrm{d}$ observation period. As previously indicated, $B$. burgdorferi initially cultured from tissue was designated passage 0 . Subsequent passages in BSK II medium were consecutively numbered. To conclusively identify spirochetes recovered from tissue as $B$. burgdorferi, 20 random isolates recovered from different sites in different rabbits were immunoblotted, probed with the mAb H3TS directed against OspA (26), kindly provided by Alan Barbour (University of Texas, San Antonio) and shown to express OspA protein of identical size as the original B31 inoculate (data not shown).

SDS-PAGE and immunoblotting. SDS-polyacrylamide slab gels were run by using the discontinuous buffer system of Laemmli (27). Samples containing $3 \times 10^{7} \mathrm{~B}$. burgdorferi were boiled for $10 \mathrm{~min}$ in final sample buffer consisting of 4\% SDS, $10 \%$ 2-mercaptoethanol, and $0.01 \%$ bromphenol blue in $125 \mathrm{mM}$ Tris buffer, pH 6.8. After electrophoresis, gels were transferred to polyvinylidene diflouride (PVDF) membranes (Millipore Corp., Bedford, MA) as described (28). For immunoblotting, PVDF membranes were incubated for $1 \mathrm{~h}$ with either mAb H3TS diluted 1:2,500, or adsorbed rabbit serum diluted 1:500; dilutions were carried out in PBS containing 5\% nonfat dry milk (Carnation Co., Los Angeles, CA) and 0.1\% Tween 20 (Sigma Chemical Co., St. Louis, MO) (MT-PBS). After washing, the blots were incubated for $1 \mathrm{~h}$ with anti-mouse immunoglobulin or Staphylococcal protein A conjugated to horseradish peroxidase (Amersham Corp., Arlington Heights, IL) diluted $1: 5,000$ or $1: 1,000$, respectively, in MTPBS. Antibody-antigen binding was detected using the enhanced chemiluminescence system of Amersham Corp. Chemilumigraphs were produced with X-AR5 film (Eastman Kodak Co., Rochester, NY). 
ELISA. Flat bottomed 96-well immunoassay plates (Immulon 4; Dynatech Laboratories, Inc., Chantilly, VA) were coated overnight at room temperature in a humidified chamber with $100 \mu \mathrm{l} \mathrm{of} 1 \mu \mathrm{g} / \mathrm{ml}$ sonicate of whole $B$. burgdorferi passage 7 in PBS. The plates were washed twice with wash buffer (0.5\% Tween 20 in PBS) and blocked for $1 \mathrm{~h}$ with BLOTTO ( $5 \%$ dry nonfat milk in PBS). After two washes with wash buffer, $100 \mu \mathrm{l}$ of twofold dilutions of rabbit serum in PBS ranging from 1:500 to 1:64,000 were added to the wells and incubated at room temperature for $2 \mathrm{~h}$. Plates were washed four times and incubated with anti-rabbit horseradish peroxidase conjugate $(1: 5,000)$ (Amersham Corp.). The plates were then washed four times and $100 \mu \mathrm{l}$ 2,2'-azinobis (3-ethyl benzthiazoline sulfonic acid) peroxidase substrate system (Kirkegaard \& Perry Laboratories, Inc., Gaithersburg, MD) was added to each well. After $30 \mathrm{~min}$, the OD of each well at $405 \mathrm{~nm}$ was measured on an automated ELISA plate reader (Titertek Multiscan MCC/340; Flow Laboratories, Baar, Switzerland). Serum from one rabbit known to be immune to reinfection (see Results) was chosen as a positive standard and run in serial dilution on each plate. A total of 21 plates was used on different days. The mean and standard deviation of each dilution were determined for this positive standard. The standard deviation was found to be no $>0.12$ at the tested dilutions.

Sera and adsorptions. Blood was obtained aseptically from the central artery of selected rabbits exhibiting different degrees of infectionderived immunity to challenge. The separated sera were frozen at $-70^{\circ} \mathrm{C}$ until ready for use. Adsorbed sera were obtained by incubating serum samples with aliquots of washed and frozen whole ATCC B31 and an acetone powder of the ATCC B31 strain prepared according to a method previously described (29). Serum was adsorbed until reactivity with the ATCC B31 strain could not be detected at 1:600 dilution on enhanced chemiluminescence Western blots. Sequential absorptions with a total of $5 \times 10^{10}$ frozen whole organisms and $6 \times 10^{10}$ acetone organism equivalents were required to adsorb $750 \mu \mathrm{l}$ of serum.

Statistical analysis. Time of appearance, duration, and diameter of the EM lesions were expressed as means and standard deviations. Analysis of ELISA data was performed as follows. Diagnostic plots of mean $\mathrm{OD}_{405}$ for each time point separately, versus the corresponding standard deviation (Box Cox analysis) implied that a square root transformation of $\mathrm{OD}_{405}$ data was required to stabilize variances (30). Mean $\mathrm{OD}_{405}$ values across various serum concentration levels were compared between the immune and susceptible groups on a square root scale. Because the measurements for the prechallenge, 14-17 d postchallenge, and 28-35 d postchallenge timepoints were on the same rabbits, a repeated measures ANOVA was used to analyze the data (30). Significant differences were accepted at the $P<0.05$ level.

\section{Results}

Induction of EM lesions in the rabbit by B. burgdorferi, strain B31. Initial studies with 36 adult New Zealand White rabbits showed that EM lesions resembling those seen in humans resulted from the ID injection of virulent strain B31 over a wide range of numbers of spirochetes passaged in vitro from 4 to 47 times. Table II shows that passages 4,8 , and 27 produced EM lesions at each site (four per rabbit) inoculated intradermally with either $10^{5}, 10^{6}$, or $10^{7}$ spirochetes; in addition, each animal inoculated with $10^{3}$ or $10^{4}$ passage 8 or 27 spirochetes developed EM at each site. Passage 47 induced EM less consistently; although EM occurred at each of the eight sites inoculated with $10^{4}$ spirochetes, EM developed at only four of eight sites injected with $10^{5}$ organisms and six of eight sites injected with $10^{3}$ organisms. Biopsies of representative EM lesions from rabbits injected with each of the above inocula were culture positive for B, burgdorferi. The avirulent ATCC B31 strain did not induce lesions, and could not be cultivated from skin biopsies taken at a time when EM appeared in rabbits infected with virulent B31.
Table II. EM after ID Inoculation of Rabbits with B. burgdorferi B31 Strain

\begin{tabular}{lccccc}
\hline & \multicolumn{5}{c}{ Inoculum * $^{\circ}$} \\
\cline { 2 - 6 } & $10^{7}$ & $10^{6}$ & $10^{5}$ & $10^{4}$ & $10^{3}$ \\
\hline & \multicolumn{5}{c}{ EM/Total sites } \\
In vitro passage & & & & & \\
4 & $8 / 8$ & $8 / 8$ & $8 / 8$ & $\mathrm{ND}$ & $\mathrm{ND}$ \\
8 & $8 / 8$ & $8 / 8$ & $8 / 8$ & $8 / 8$ & $8 / 8$ \\
27 & $8 / 8$ & $8 / 8$ & $8 / 8$ & $8 / 8$ & $8 / 8$ \\
47 & $8 / 8$ & $8 / 8$ & $4 / 8$ & $8 / 8$ & $6 / 8$ \\
ATCC B31 & & & & & \\
$\quad$ Avirulent control & $0 / 8$ & $\mathrm{ND}$ & $\mathrm{ND}$ & $\mathrm{ND}$ & $\mathrm{ND}$ \\
& & & & & \\
\hline
\end{tabular}

* Two rabbits were injected at four sites for each inoculum tested. ND, not done.

Each of the 138 EM lesions resembled human EM. They appeared as either uniformly erythematous, banded, or target patterns; individual rabbits showed only one type of lesion pattern at each of the injection sites (Fig. 1). Lesions appeared in $6 \pm 1 \mathrm{~d}$, with a range of 3-8 d. They expanded significantly, at their peak averaged $27.8 \pm 20.5 \mathrm{~mm}$ in diameter with a range of $7.5-79 \mathrm{~mm}$, and remained visible for $5 \pm 2 \mathrm{~d}$. No differences in time to lesion appearance, lesion pattern, or size was observed among rabbits injected with numbers of spirochetes ranging from $10^{3}$ to $10^{7}$.

Further studies with 16 additional rabbits confirmed the consistency of EM production. Each of 108 sites injected with $10^{7}$ B31 passage 4 per site developed typical EM lesions. These lesions were similar in incubation period $(5 \pm 1 \mathrm{~d})$, size $(28.1 \pm 9.7 \mathrm{~mm})$, and persistence $(4 \pm 3 \mathrm{~d})$ of the lesions observed in the initial experiment. Further, representative lesions from each rabbit were also culture positive.

Histopathology of EM in the rabbit. Histopathological studies were conducted on representative EM skin biopsy sections from rabbits infected with the B31 strain of $B$. burgdorferi. Sections of skin from the lesion were stained with hematoxylin and eosin and examined microscopically. Examination at low power showed an intact keratinizing epithelium overlying dermis with infiltrating lymphocytic inflammatory cells in both the superficial and deep dermal layers (Fig. 2). The infiltrate was diffuse in the superficial dermis. Inflammatory aggregates were more pronounced in the deep dermis, and deep inflammatory aggregates were especially prominent around adnexal structures, vessels, and small nerves. These deep inflammatory infiltrates produced an appearance of perivascular cuffing with extension of inflammation between and surrounding adjacent connective tissue bundles. When examined by high power, the infiltrate was a mixed lymphocyte population with occasional macrophages and plasma cells (Fig. 3). Lymphocytes extended around small vessels leaving the endothelium largely intact. In areas of resolution, pyknosis and karyorrhexis of the inflammatory cells were apparent, without marked residual damage to surrounding structures.

Ability of additional $B$. burgdorferi strains to induce EM. Strains N40, SH-2-82, 297, HB19, CA-2-87, and ECM-NY-86 were injected ID into four rabbits using $10^{7}$ organisms per injection site. As shown in Table III, five of the six strains 


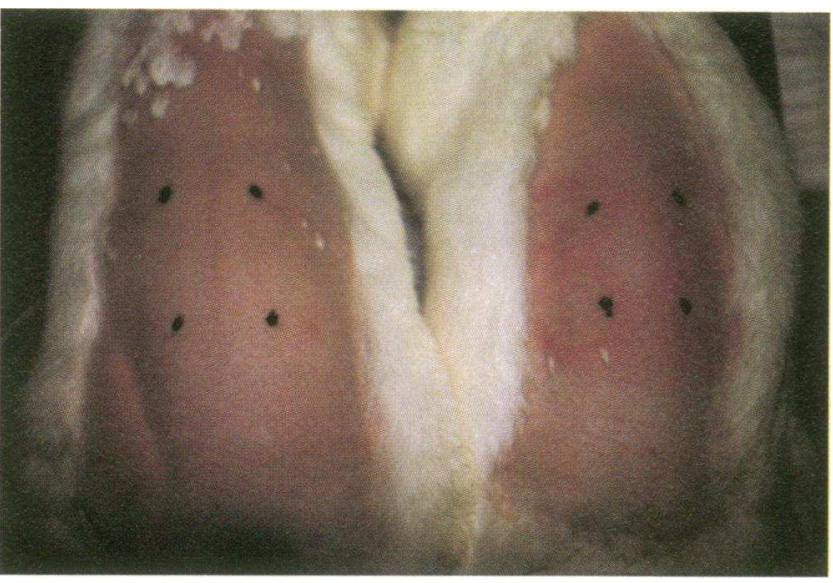

Figure 1. Erythema migrans in the New Zealand White rabbit. (Right) Typical EM target lesions at each of four sites $9 \mathrm{~d}$ after ID injection with $10^{7}$ B. burgdorferi, B31 strain passage 4. (Left) Note the absence of lesions following ID injection of $10^{7}$ avirulent B. burgdorferi, B31 strain (ATCC).

produced culture-positive EM lesions at every site, in either erythematous or target patterns, indistinguishable from those seen with virulent strain B31. In contrast, only 11 of the 24 sites injected with the ECM-NY-86 strain developed EM lesions. In all cases including ECM-NY-86, lesions appeared in $5 \pm 1 \mathrm{~d}$

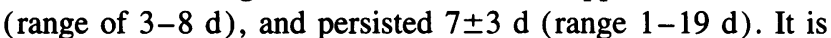
important to note that representative sites inoculated with ECMNY-86 at which lesions failed to develop were also culture positive.

Dissemination of strain B31 after intradermal injection. Tissues from 12 rabbits injected ID with $4 \times 10^{7} \mathrm{~B} 31$ passage 4 were cultured to determine the extent of visceral dissemination; two rabbits were killed at the timepoints specified in Table IV $A$. B. burgdorferi was cultured from blood, spleen, liver, and heart at $24 \mathrm{~h}$ but could not be cultured from these tissues at later timepoints. Infection of the ear and popliteal nodes were first detected at 1 wk after infection and in the spinal cord at the 2-wk timepoint. Infection remained demonstrable at the 3-

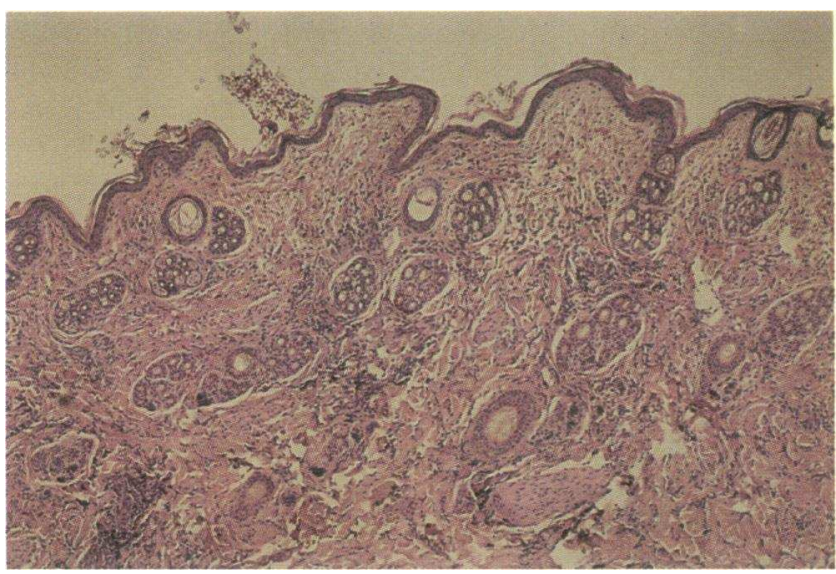

Figure 2. Histopathology of rabbit erythema migrans. At $\times 40$ stained sections show diffuse lymphocytic infiltration of the superficial dermis. Lymphocytic aggregates are more prominent in deep dermal layers, especially around vessels and adenexa, producing an appearance of inflammatory cuffing.

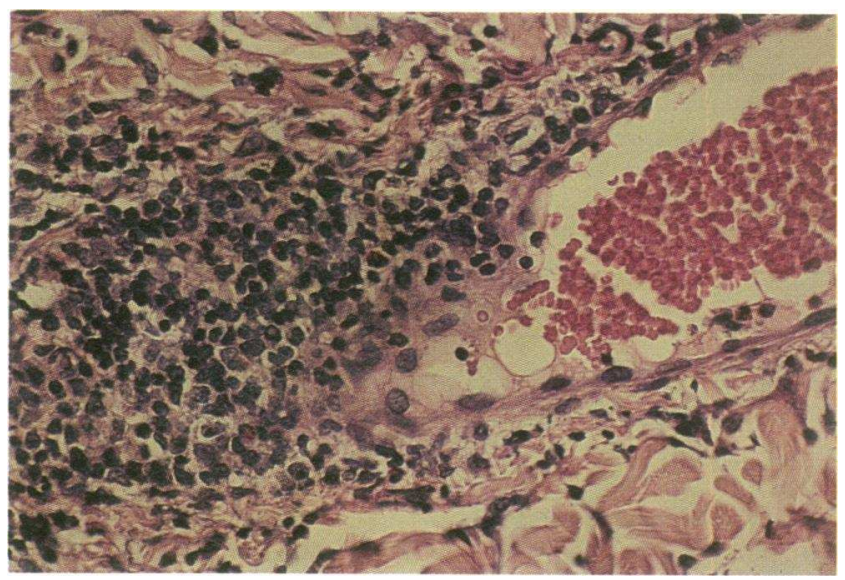

Figure 3. Histopathology of rabbit erythema migrans. At $\times 250$, the mixed cellularity of the lymphocytic infiltrate in the deep dermis is evident, with occasional plasma cells and macrophages. The infiltrate is adjacent to a small vessel with intact endothelium.

and 4-wk timepoints. Infection of the conjunctiva, stifle joint tissue, and synovial fluid was not determined before the 2-wk timepoint. However, each of these tissues was culture positive at $2 \mathrm{wk}$ and joint tissue remained positive at 3 and $4 \mathrm{wk}$. Lung, kidney, bladder, cerebral cortex tissue, and cerebrospinal fluid was cultured at each timepoint and found to be consistently culture negative (data not shown). Rabbits were examined grossly for evidence of arthritis and found to have none. Hematoxylin and eosin-stained sections made from representative culture positive joints and spinal cords have not shown evidence of infiltrating cells or other evidence of inflammation.

The extent of dissemination to the lymph nodes, joint tissue, and spinal cord was also determined by culture during an 8-wk period after the intradermal inoculation of 10 rabbits with 6 $\times 10^{3}$ B31 passage 4 (Table IV $B$ ). At 2 wk after inoculation,

Table III. Production of EM after ID Inoculation of Rabbits with Seven Strains of $B$. burgdorferi

\begin{tabular}{lccc}
\hline & \multicolumn{3}{c}{ Lesions } \\
\cline { 2 - 4 } \multicolumn{1}{c}{ Strain* } & EM/sites & Time of appearance & Duration \\
\hline & & $d^{\ddagger}$ & $d^{5}$ \\
B31 & $24 / 24$ & $3-8$ & $1-9$ \\
N40 & $24 / 24$ & $4-5$ & $5-19$ \\
SH-2-82 & $24 / 24$ & $4-7$ & $5-15$ \\
HB19 & $24 / 24$ & $4-7$ & $2-7$ \\
297 & $24 / 24$ & $4-5$ & $1-6$ \\
Ca-2-87 & $24 / 24$ & $4-6$ & $5-9$ \\
ECM-NY-86 & $11 / 24$ & 5 & $5-7$ \\
Heat-killed & $0 / 8$ & N/A & N/A \\
ATCC Avirulent B31 & $0 / 8$ & N/A & N/A \\
& & & \\
\hline
\end{tabular}

* Four rabbits were inoculated per strain with $10^{7}$ organisms per site. Controls include 2 animals inoculated with $2 \times 10^{7}$ heat-killed organisms $\left(56^{\circ} \mathrm{C}\right.$ for $45 \mathrm{~min}$ ) from each strain and two animals which were inoculated with $4 \times 10^{7}$ ATCC B31. Bladder isolates from infected mice were used at in vitro passage 1 . ${ }^{\ddagger}$ Mean $=5 \pm 1 ;{ }^{8}$ mean $=7 \pm 3$. N/A, not applicable. 
Table IV. Dissemination of B. burgdorferi B31 Passage 4 after ID Inoculation

(A) Intradermal inoculation of a total of $4 \times 10^{7}$ B. burgdorferi B31

\begin{tabular}{lcccccc}
\multicolumn{1}{c}{ Tissues } & 3 and $5 \mathrm{~h}$ & $24 \mathrm{~h}$ & 1 wk & 2 wk & 3 wk & 4 wk \\
\hline Skin & $1 / 2$ & $2 / 2$ & $2 / 2$ & $2 / 2$ & $2 / 2$ & $2 / 2$ \\
Ear & $0 / 2$ & $0 / 2$ & $1 / 2$ & $1 / 2$ & $1 / 2$ & $1 / 2$ \\
Blood & $0 / 2$ & $0 / 2$ & $0 / 2$ & $0 / 2$ & $0 / 2$ & $0 / 2$ \\
Spleen & $0 / 2$ & $2 / 2$ & $0 / 2$ & $0 / 2$ & $0 / 2$ & $0 / 2$ \\
Liver & $0 / 2$ & $2 / 2$ & $0 / 2$ & $0 / 2$ & $0 / 2$ & $0 / 2$ \\
Heart & $0 / 2$ & $1 / 2$ & $0 / 2$ & $0 / 2$ & $0 / 2$ & $0 / 2$ \\
Popliteal nodes & $0 / 4$ & $0 / 4$ & $1 / 2$ & $1 / 2$ & $2 / 2$ & $1 / 2$ \\
Stifle joint tissue & ND & ND & ND & $1 / 1$ & $1 / 2$ & $1 / 2$ \\
Synovial fluid & ND & ND & ND & $1 / 2$ & $0 / 2$ & $0 / 2$ \\
Conjunctiva & ND & ND & ND & $2 / 2$ & $0 / 2$ & $0 / 2$ \\
Spinal cord & $0 / 4$ & $0 / 4$ & $2 / 2$ & $2 / 2$ & $2 / 2$ & $2 / 2$
\end{tabular}

(B) Intradermal inoculation of a total of $6 \times 10^{3}$ B. burgdorferi B31

\begin{tabular}{lcccccc}
\multicolumn{1}{c}{ Tissues } & 1 wk & 2 wk & 3 wk & 4 wk & 5 wk & 8 wk \\
\hline Skin & $9 / 10$ & $3 / 3$ & $6 / 7$ & $3 / 3$ & $3 / 4$ & $1 / 4$ \\
Popliteal nodes & ND & $2 / 3$ & ND & $1 / 3$ & ND & $0 / 4$ \\
Stifle joint tissue & ND & $3 / 3$ & ND & $2 / 3$ & ND & $0 / 4$ \\
Spinal cord & ND & $1 / 3$ & ND & $0 / 3$ & ND & $0 / 4$
\end{tabular}

Data is expressed as No. of rabbits/total No. of rabbits. ND, not done.

infection of the nodes, joints, and spinal cord was demonstrable. At the 4-wk timepoint the popliteal nodes and joint tissue were still culture positive. At $8 \mathrm{wk}$ after inoculation, disseminated infection was not demonstrable in any of these tissues.

Clearance of skin and visceral infection with $B$. burgdorferi $B 31$. In an effort to provide additional insight into the time at which dermal and visceral infection clears, 30 rabbits were infected with $6 \times 10^{7}$ B. burgdorferi B31 passage 1 and monitored at 2-wk intervals. Skin biopsies were obtained from rabbits at $1,4,6,8,10$, and $12 \mathrm{wk}$ from a site in proximity to the injection site. At 4, 6, 8, 10, and $12 \mathrm{wk}$, groups of three rabbits were killed for the determination of visceral infection. As shown in Fig. 4, a progressive decrease of positive skin biopsies was observed from wk 4 to 12 , at which time all of the animals had cleared the infection; the mean duration of infection was $6.7 \pm 1.4$ wk. Dissemination occurred 4-8 wk after infection but was not demonstrable at the 10- and 12-wk timepoints (data not shown). More evidence for the clearance of visceral infection is shown in the experiment described above (Table IV $B$ ). In this experiment clearance of dissemination following the ID inoculation of $6 \times 10^{3}$ B31 passage 4 occurred by 8 wk after inoculation.

Dissemination of additional B. burgdorferi strains after intradermal injection. Dissemination studies similar to those conducted for the B31 strain were initiated with six additional strains utilizing two rabbits per strain. As shown in Table V, 4 wk after ID inoculation, at a time when the skin biopsy cultures were still positive, three of the six strains showed dissemination to the popliteal nodes and spinal cord; joint tissue of one of these strains and one additional strain were also culture positive. Strains CA-2-87 and ECM-NY-86 were not cultured from the viscera.

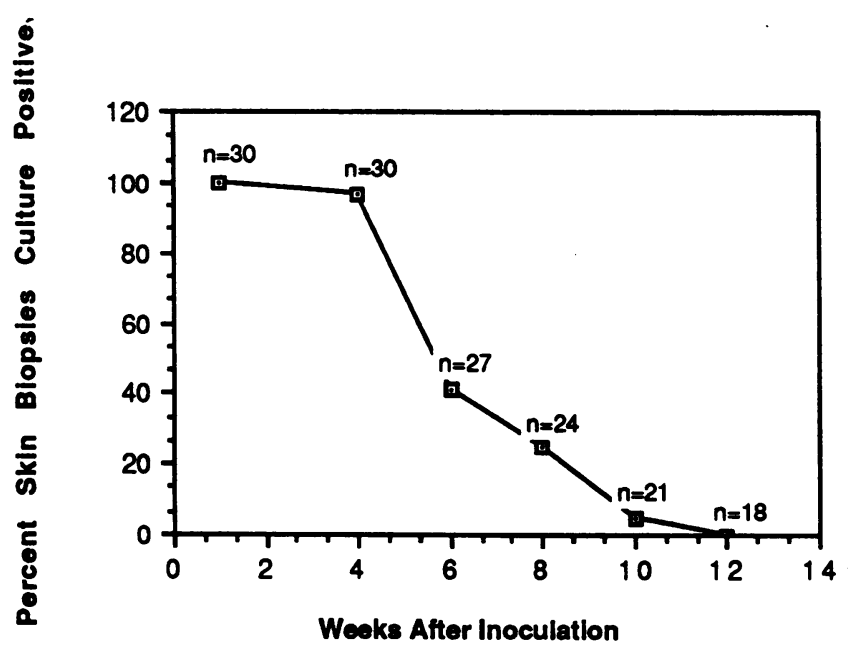

Figure 4. Clearance of B. burgdorferi B31 from the skin after intradermal infection. Skin biopsies were obtained at each timepoint and cultured in BSK II medium as described in the text. - - , percentage of culturepositive rabbits.

Development of infection-derived immunity to strain B31. A study was designed to determine whether rabbits which had cleared EM and dermal infection with the B31 strain were immune to reinfection (Table VI). A total of 26 rabbits were first injected at four intradermal sites with B31 passage 4, 8, 27, or 47 utilizing log dilutions for each passage ranging from $10^{3}$ to $10^{7}$ organisms per site. As controls, six rabbits were similarly injected with $4 \times 10^{7}$ avirulent ATCC B31 strain or B31 strain passage four heat killed at $56^{\circ} \mathrm{C}$ for $45 \mathrm{~min}$. Approximately $5 \mathrm{mo}$ later, rabbits were challenged ID at two sites for each dilution of $10^{5}, 10^{6}$, and $10^{7}$ B. burgdorferi. Each of the six rabbits initially injected with ATCC B31 strain or heat-killed B31 passage 4 were shown to be susceptible upon challenge as measured by the development of culture-positive EM. In contrast, each of the seven rabbits initially infected with the log dilutions ranging from $4 \times 10^{3}$ to $4 \times 10^{7} \mathrm{~B} 31$ passage 4 exhibited complete protection against $\mathrm{EM}$ and dermal infection upon challenge with the three concentrations of homologous $B$. burgdorferi. This was evidenced by the absence of lesion development at each of the 42 sites inoculated and the inability to culture $B$. burgdorf-

Table V. Dissemination of Six Strains of B. burgdorferi $4 \mathrm{Wk}$ after Intradermal Inoculation

\begin{tabular}{lllllll}
\hline & \multicolumn{6}{c}{ Strain } \\
\cline { 2 - 7 } & N40 & SH-2-82 & HB19 & 297 & CA-2-87 & ECM-NY-86 \\
\hline Tissues & & & & & & \\
Skin & $2 / 2$ & $2 / 2$ & $2 / 2$ & $2 / 2$ & $2 / 2$ & $2 / 2$ \\
Popliteal nodes & $2 / 2$ & $1 / 2$ & $0 / 2$ & $1 / 2$ & $0 / 2$ & $0 / 2$ \\
Stifle joint tissue & $1 / 2$ & $0 / 2$ & $2 / 2$ & $0 / 2$ & $0 / 2$ & $0 / 2$ \\
Synovial fluid & $0 / 2$ & $0 / 2$ & $0 / 2$ & $0 / 2$ & $0 / 2$ & $0 / 2$ \\
Spinal cord & $2 / 2$ & $1 / 2$ & $0 / 2$ & $1 / 2$ & $0 / 2$ & $0 / 2$ \\
& & & & & & \\
\hline
\end{tabular}

For all strains two rabbits were inoculated at each of eight sites with $10^{7}$ organisms per site. Strains used were obtained from skin biopsy cultures of previously infected rabbits and used at in vitro passage 4 . Data are expressed as positive rabbits/total rabbits. 
Table VI. Homologous Challenge of Rabbits Infected $\sim 5$ Mo Previously with $B$. burgdorferi B31

\begin{tabular}{|c|c|c|c|c|c|}
\hline \multirow[b]{2}{*}{ Initial injection } & & \multicolumn{3}{|c|}{ Challenge dose* } & \multirow[b]{2}{*}{ Culture results } \\
\hline & & $10^{7}$ & $10^{6}$ & $10^{5}$ & \\
\hline B. burgdorferi & Inoculum & \multicolumn{2}{|c|}{ EM/total sites } & & at day $5-7^{\ddagger}$ \\
\hline Noninfectious & N/A & $6 / 6$ & $6 / 6$ & $6 / 6$ & + \\
\hline Heat-killed & $4 \times 10^{7}$ & $3 / 4$ & $4 / 4$ & $4 / 4$ & + \\
\hline Lab strain & $4 \times 10^{7}$ & $7 / 8$ & $6 / 8$ & $4 / 8$ & + \\
\hline \multirow[t]{5}{*}{ Passage 4} & $4 \times 10^{7}$ & $0 / 4$ & $0 / 4$ & $0 / 4$ & - \\
\hline & $4 \times 10^{6}$ & $0 / 2$ & $0 / 2$ & $0 / 2$ & - \\
\hline & $4 \times 10^{5}$ & $0 / 2$ & $0 / 2$ & $0 / 2$ & - \\
\hline & $4 \times 10^{4}$ & $0 / 2$ & $0 / 2$ & $0 / 2$ & - \\
\hline & $4 \times 10^{3}$ & $0 / 4$ & $0 / 4$ & $0 / 4$ & - \\
\hline \multirow[t]{5}{*}{ Passage 8} & $4 \times 10^{7}$ & $0 / 2$ & $0 / 2$ & $0 / 2$ & - \\
\hline & $4 \times 10^{6}$ & $2 / 4$ & $0 / 2$ & $0 / 2$ & - \\
\hline & $4 \times 10^{5}$ & $1 / 2$ & $0 / 2$ & $0 / 2$ & - \\
\hline & $4 \times 10^{4}$ & $0 / 4$ & $0 / 4$ & $0 / 4$ & - \\
\hline & $4 \times 10^{3}$ & $3 / 4$ & $2 / 4$ & $3 / 4$ & - \\
\hline \multirow[t]{5}{*}{ Passage 27} & $4 \times 10^{7}$ & $2 / 4$ & $2 / 4$ & $2 / 4$ & - \\
\hline & $4 \times 10^{6}$ & $2 / 2$ & $0 / 2$ & $0 / 2$ & - \\
\hline & $4 \times 10^{5}$ & $2 / 4$ & $2 / 4$ & $1 / 4$ & - \\
\hline & $4 \times 10^{4}$ & $0 / 2$ & $0 / 2$ & $0 / 2$ & - \\
\hline & $4 \times 10^{3}$ & $2 / 4$ & $2 / 4$ & $2 / 4$ & - \\
\hline \multirow[t]{3}{*}{ Passage 47} & $4 \times 10^{7}$ & $2 / 2$ & $0 / 2$ & $0 / 2$ & + \\
\hline & $4 \times 10^{4}$ & $0 / 2$ & $0 / 2$ & $0 / 2$ & + \\
\hline & $4 \times 10^{3}$ & $2 / 2$ & $2 / 2$ & $2 / 2$ & + \\
\hline
\end{tabular}

* Rabbits were challenged with passage 4 B31. EM lesions were developed at 5-7 d after challenge. ${ }^{\ddagger}$ Negative biopsied skin cultures obtained at $5-7 \mathrm{~d}$ were repeated and found negative at $18,32,46,67$, and $81 \mathrm{~d}$ after challenge. N/A, not applicable.

eri from representative sites. It is striking to note that infection with as few as $4 \times 10^{3}$ passage $4 \mathrm{~B} 31$ was fully efficacious in inducing acquired resistance to reinfection. The eight rabbits infected initially with B31 passage 8 and the eight rabbits infected initially with B31 passage 27 exhibited partial but not complete protection as evidenced by development of EM at 30 of 96 sites challenged with $10^{5}, 10^{6}$, or $10^{7} \mathrm{~B} 31$ passage 4 but uniform culture negativity of representative biopsied lesions. The three rabbits initially infected with $4 \times 10^{3}, 4 \times 10^{4}$, or $4 \times 10^{7}$ B31 passage 47 exhibited the least protection against challenge, inasmuch as skin biopsy cultures were positive at all sites and EM developed at 8 of the 18 challenge sites. These observations indicate that the ability of infection with strain B31 to induce complete immunity against EM lesion development and dermal infection is progressively lost upon continuous in vitro passage.

Development of infection-derived immunity to additional $B$. burgdorferi strains. Using the experimental design described in the preceding section, the degree of protective immunity resulting from untreated infection was determined for six additional $B$. burgdorferi strains. Rabbits were infected ID with each strain indicated in Table VII by injection of $1 \times 10^{7}$ organisms passage 4 at each of four sites. Approximately 5 mo later, after representative skin biopsy cultures were negative,
Table VII. Homologous Intradermal Challenge of Rabbits Infected 5 Mo Previously with $4 \times 10^{7}$ Low Passaged Strains of $B$. burgdorferi

\begin{tabular}{lccc}
\hline \multicolumn{1}{c}{ Strain } & $\begin{array}{c}\text { EM/ } \\
\text { total sites }\end{array}$ & $\begin{array}{c}\text { Skin biopsy } \\
\text { cultures: } \\
\text { positive rabbits }\end{array}$ & $\begin{array}{c}\text { Visceral } \\
\text { cultures: } \\
\text { positive rabbits }\end{array}$ \\
\hline B31 & $0 / 16$ & $0 / 2$ & $0 / 2$ \\
N40 & $0 / 16$ & $0 / 2$ & $0 / 2$ \\
SH-2-82 & $0 / 16$ & $0 / 2$ & $0 / 2$ \\
HB19 & $8 / 16$ & $0 / 2$ & $0 / 2$ \\
297 & $8 / 16$ & $0 / 2$ & $0 / 2$ \\
CA-2-87 & $0 / 16$ & $2 / 2$ & $0 / 2$ \\
ECM-NY-86 & $0 / 16$ & $0 / 2$ & $0 / 2$ \\
Controls & & & \\
Heat killed & $16 / 16$ & $2 / 2$ & $1 / 2$ \\
ATCC Avirulent B31 & $16 / 16$ & $2 / 2$ & $1 / 2$ \\
Noninfected & $99 / 112 *$ & $14 / 14$ & $8 / 14^{\ddagger}$ \\
& & & \\
\hline
\end{tabular}

Two rabbits were challenged at each of eight sites with $10^{7}$ organisms/ site. Strains used for challenge were obtained from skin biopsy cultures of previously infected rabbits and used at in vitro passage 4. Data shown for punch biopsy cultures were obtained at 7-11 d after challenge. Similar data were obtained at days 21 and 28. Controls include the low passage B31 challenge of those animals previously inoculated with either $2 \times 10^{7}$ heat-killed organisms from each strain or given $4 \times 10^{7}$ avirulent ATCC B31, and inoculation of two noninfected animals for each strain. Visceral cultures examined in each rabbit were spinal cord, popliteal lymph nodes, and stifle joint tissue. $* 13$ of 16 sites inoculated with the ECM-NY-86 strain failed to develop lesions. ${ }^{\ddagger}$ See Table V.

each of the rabbits and two naive controls per strain were challenged ID with $1 \times 10^{7}$ passage 4 of the same strain at eight sites. Skin biopsies at representative sites were performed at days 7,21 , and $28 \mathrm{~d}$ postchallenge; dissemination to popliteal lymph nodes, stifle joints, and spinal cord, was determined at 28 days postchallenge.

As shown in Table VII, complete homologous protection against both EM and dissemination occurred with three strains, B31, N40, and SH-2-82, as measured by the failure to develop EM lesions (Fig. 5) and failure to culture organisms from skin and tissues. In contrast, the two naive controls exhibited EM at every site, as well as disseminated infection. Further, each of two rabbit controls injected ID 5 mo earlier with $4 \times 10^{7}$ heatkilled B31 or the avirulent ATCC B31 strain developed lesions at each of 16 inoculated sites upon challenge with the homologous B31 strain; dissemination was also demonstrable.

Partial or complete homologous protection against EM and/ or dissemination was observed among the remaining four strains. Two strains, HB19 and 297, did not completely protect against the development of EM. In each case one of the two challenged animals developed EM at each of the inoculated sites. It is interesting to note that the EM lesions in these animals were culture negative at all timepoints tested and, in contrast to the naive controls, dissemination in these animals was not demonstrable. Strain CA-2-87 did not develop EM upon challenge but organisms were recovered from skin biopsies from both rabbits. Dissemination was not demonstrated for either the challenge or the control animals.

ECM-NY-86 was the only strain in which EM lesions failed to develop at 13 of 16 sites on the uninfected control animals, 


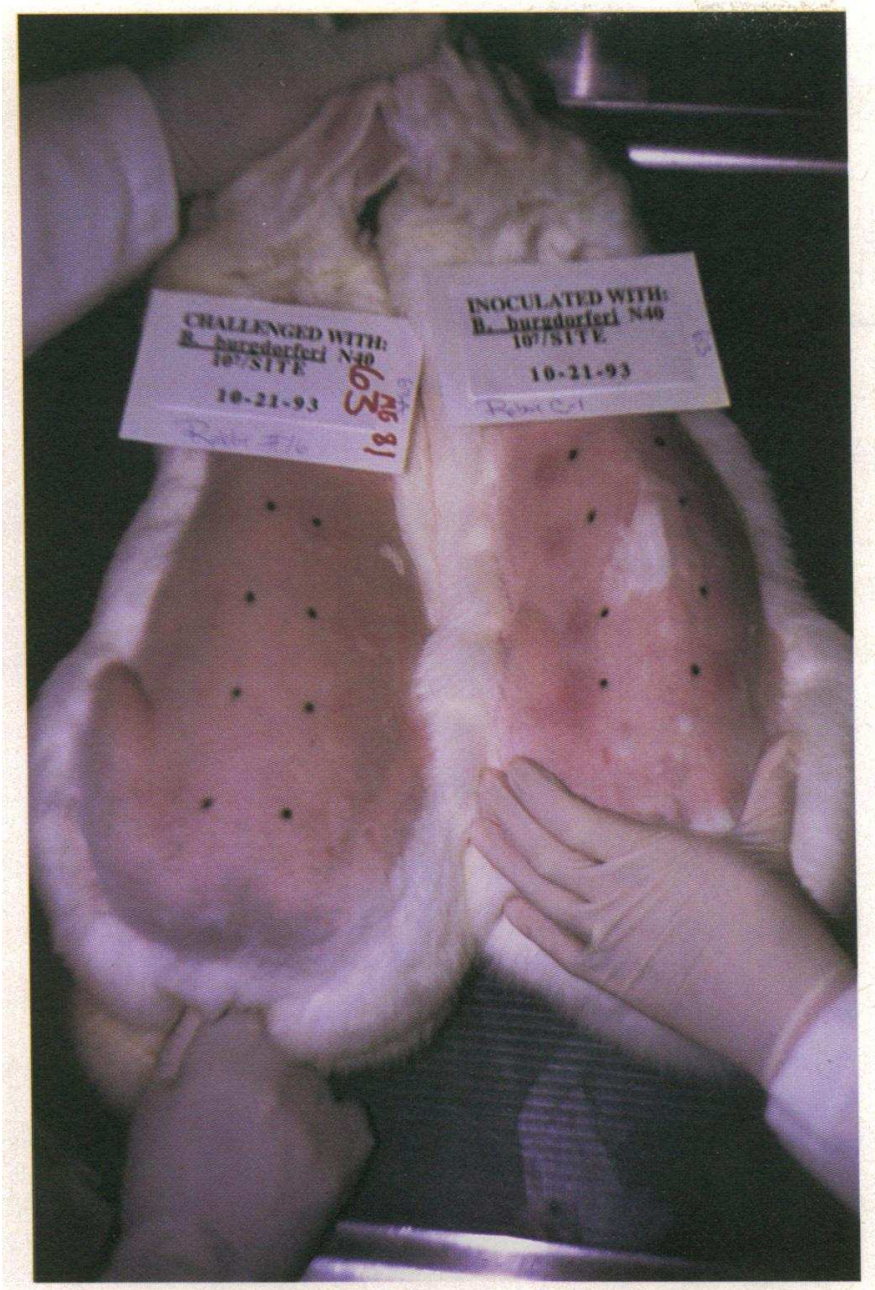

Figure 5. Infection-derived immunity to intradermal challenge. Rabbits initially infected ID with $4 \times 10^{7}$ B. burgdorferi, N40 strain, and challenged 5 mo later with $10^{7} \mathrm{~N} 40$ at each of eight sites, did not develop $\mathrm{EM}$, in contrast to previously uninfected controls.

although 10 of 10 skin biopsy cultures were positive at the 7-, 21-, and 28-d timepoints; dissemination was not observed in these control animals. Rabbits challenged with ECM-NY-86 failed to develop lesions or dissemination. However, the failure to produce lesions at every site as well as the failure to observe dissemination in the control animals precludes any conclusion with respect to the ability of the ECM-NY-86 strain to produce infection-derived immunity.

Antibody response to infection and challenge. Potential quantitative differences in the antibody response of immune versus susceptible rabbits after infection and challenge with $B$. burgdorferi were determined by ELISA. The tests were conducted on prechallenge, 14-17 d postchallenge, and 28-35 d postchallenge sera obtained from 18 completely immune rabbits, 2 susceptible rabbits initially infected with passage 47 B31, and 6 control susceptible rabbits initially infected with the ATCC B31 or heat-killed low passage organisms. As shown in Fig. 6, although each rabbit generated a primary and anamnestic antibody response to the whole organism, no significant correlation was observed between the titers and the status of immunity.

Immune serum defines antigens uniquely associated with virulent strain B31 and related to induction of infection-derived immunity. We hypothesized that the in vitro passage related differences in the ability of strain B31 to infect, to produce $E M$, and to induce different degrees of protective immunity described in the preceding sections might correspond to antigenic changes in $B$. burgdorferi. Norris has previously reported that 28- (OspD) and 35-kD lipoproteins are found in virulent strain B31, but are absent in the avirulent ATCC B31 strain (31). To test if virulent B31 was antigenically distinct from less virulent and avirulent B31 (Table VI), serum from a rabbit completely immune to infection with virulent strain B31 was adsorbed extensively with avirulent ATCC B31 strain as described in Methods. The immunoblot analysis shown in Fig. 7 $B$ demonstrated that after extensive adsorbtions, only 19- and 35-kD proteins of avirulent ATCC B31 strain bound antibody (lane 4). In contrast, 70-, 55-, 35-, 32-, 28-, 19-, 18-, 17.5-, and $15.5-\mathrm{kD}$ antigens of $\mathrm{B} 31$ passage 1 were detected. B31 passage 27 lacked the 70-, 55-, and 17.5-kD antigens found in passage 1 but retained reactivity with the 35-, 32-, 28-, 19-, 18and $15.5-\mathrm{kD}$ proteins. Adsorbed antisera showed reactivity with antigens $38,35,33,29,27,17.5$, and $17 \mathrm{kD}$ of the passage 47 isolate yet when compared to immunoblot protein profiles of the passage 1 and 27 isolates differed significantly in the intensity of the reactivity. These findings may relate to the differences in infectivity, ability to induce EM, and ability to induce protective immunity exhibited by different passages of $B$. burgdorferi strain B31, as shown in Tables II and VII. While the adsorbed antiserum used for these studies was derived from a single immune rabbit, we have obtained identical results using adsorbed serum obtained from a second immune rabbit when tested against low passage and the ATCC B31 strains.

It should be noted that none of the bands recognized with the adsorbed immune serum are OspA, as the OspA content of each of the passages of B31 was indistinguishable, equivalent numbers of spirochetes $\left(5 \times 10^{7}\right)$ were loaded per lane (Fig. $7 \mathrm{~A}$ ), and clearly no antigen is recognized in the molecular mass range of $31 \mathrm{kD}$ in the ATCC B31 (Fig. $7 \mathrm{~B}$, lane 4). In addition, the adsorbed antiserum did not bind up to $5 \mu \mathrm{g}$ of OspA on a two dimensional immunoblot (Skare, J. T., E. S. Shang, D. M. Foley, D. R. Blanco, C. I. Champion, T. Mirzabekov, Y. Sokolov, B. L. Kagan, J. N. Miller, and M. A. Lovett, script in preparation).

\section{Discussion}

This report demonstrates that infection of the New Zealand White rabbit with $B$. burgdorferi has unique features for the study of pathogenesis and immunity in Lyme borreliosis. The rabbit is very sensitive to infection with $B$. burgdorferi; intradermal injection of $10^{3} \mathrm{~B}$. burgdorferi low passage strain B31 is sufficient to reproducibly induce lesions characteristic of EM and chronic skin and visceral infection, including the knee joints and the central nervous system. Development of immunity to reinfection is a striking feature of the rabbit model. Within 3 mo after initial infection, both skin and visceral infection are cleared. $5 \mathrm{mo}$ after the initial intradermal injection of $4 \times 10^{3}$ passage 4 , strain $B 31$, subsequent challenge with $4 \times 10^{7}$ passage 4 resulted in no dermal evidence of reinfection. It is interesting that progressive in vitro passage of strain B31 (to passage 47) does little to diminish its infectivity or ability to induce $\mathrm{EM}$, but abrogates its ability to protect against reinfection. Each of these key facets of the model will be considered individually 


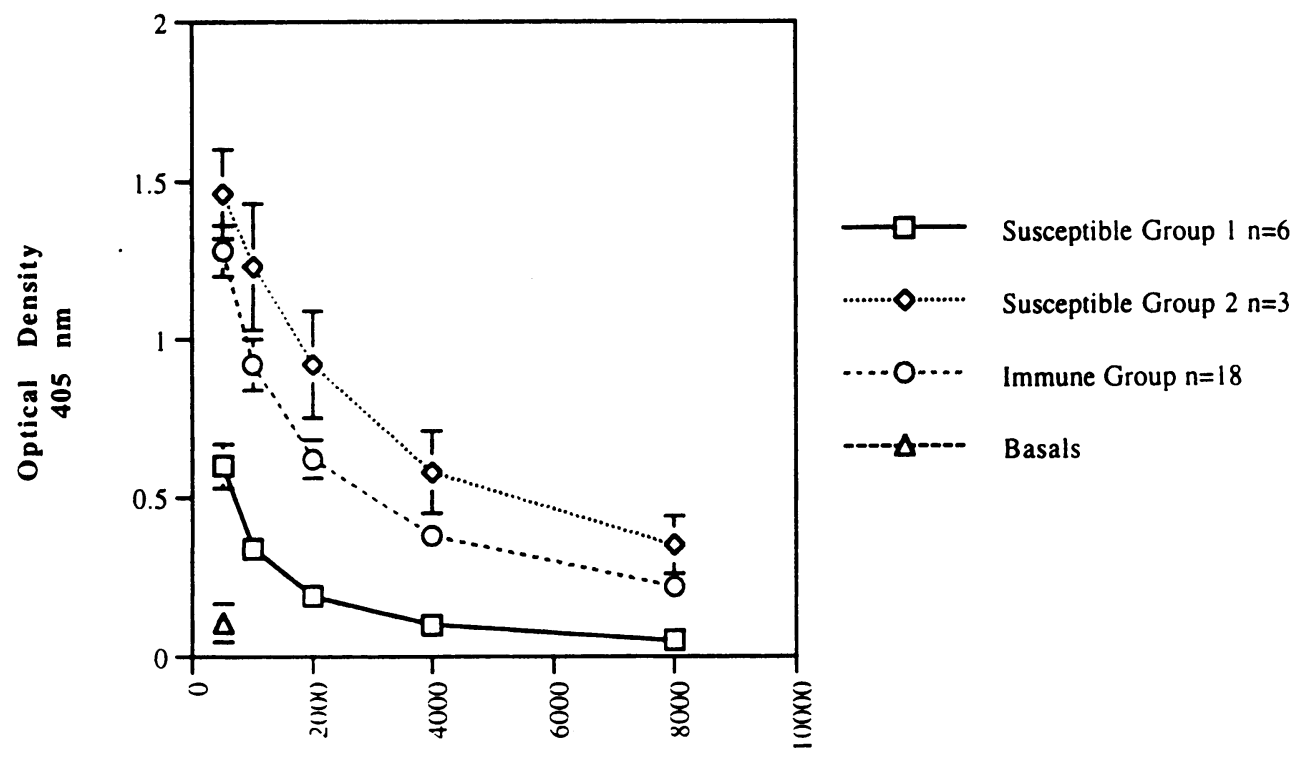

Serum Dilutions

B

B

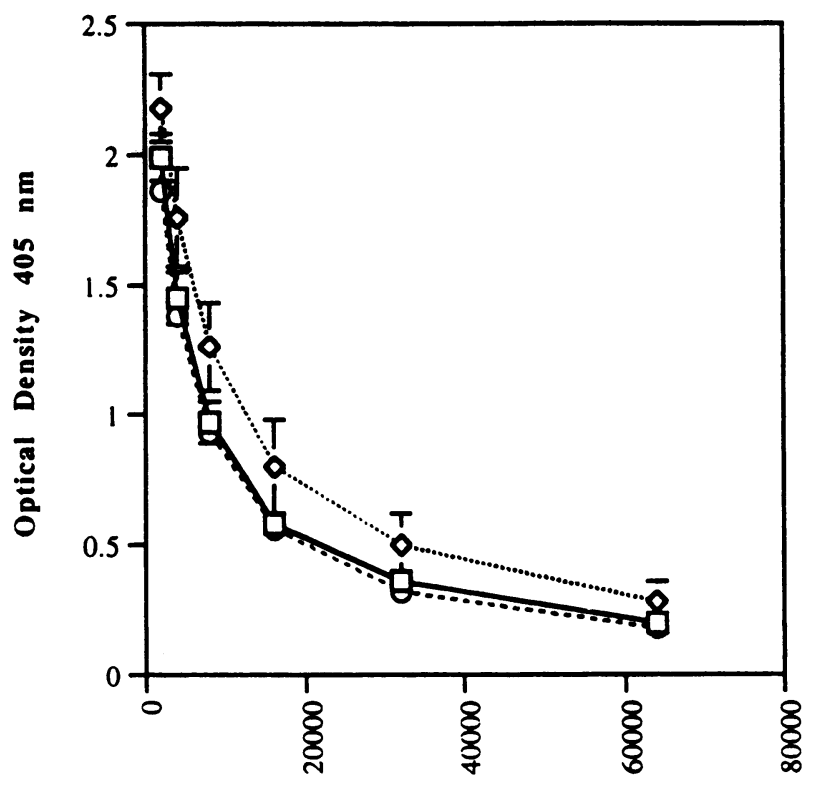

Serum Dilutions

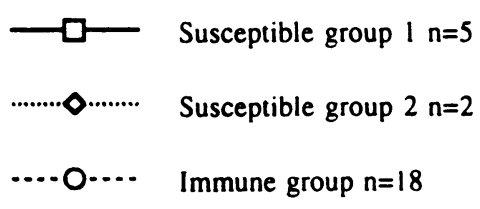

Figure 6. ELISA showing antibody titers of rabbits susceptible or immune to intradermal infection with $B$. burgdorferi. Susceptible group 1 represents ATCC and heat inactivated inoculated controls and susceptible group 2 represent animals which were inoculated with passage 47. (A) Prechallenge $(B)$ 14-17 d postchal lenge $(C)$ 28-35 d postchallenge. Error bars reflect standard error. No significant difference between immune and susceptible animals was noted for any timepoint. in the context of previous studies of experimental Lyme borreliosis.

Although the production of EM and dissemination in the rhesus monkey has been reported (18), the demonstration of $\mathrm{EM}$ in small animals such as rats $(9,10)$, mice $(11,12)$, hamsters (13-15), gerbils (16), and guinea pigs (17) has not been described despite the susceptibility of these animals to chronic infection with $B$. burgdorferi. Prior attempts to repro- ducibly induce EM in the rabbit have been unsuccessful. Although erythematous lesions have occasionally been observed before this study, B. burgdorferi has not been cultured from putative EM, nor has the characteristic histopathology of EM been demonstrated. Burgdorfer and his colleagues (20) described the appearance of annular, erythematous lesions in New Zealand White rabbits after being fed upon by Ixodes scapularis infected with $B$. burgdorferi. However, these lesions rarely ap- 


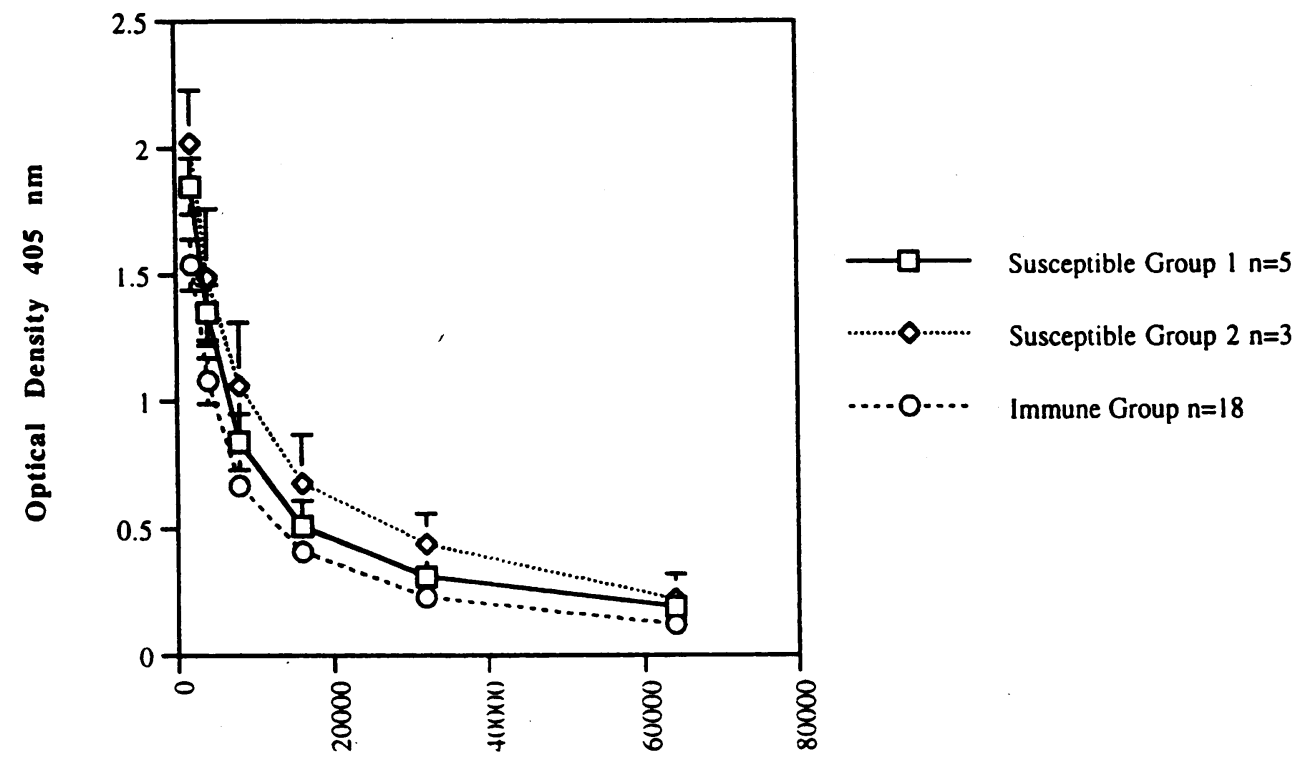

Serum Dilutions

Figure 6 (Continued)
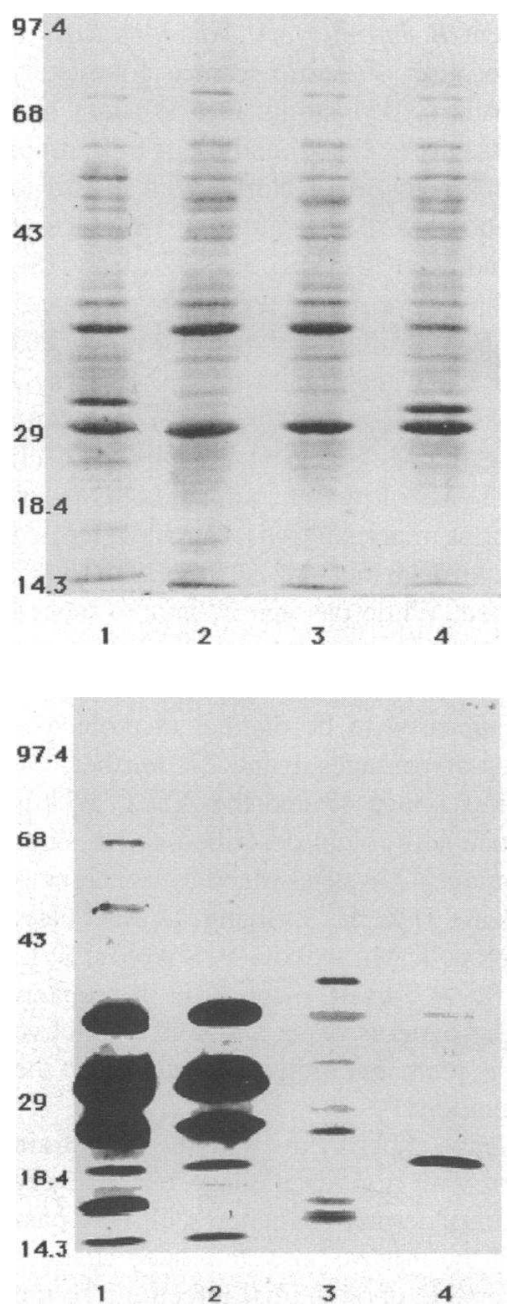

Figure 7. Virulent strain-associated antigens (va) of $B$. burgdorferi $\mathrm{B} 31$. A $5-15 \%$ gradient gel was run and transferred to PVDF. Each lane of this immunoblot contains $5 \times 10^{7} \mathrm{~B} 31$ of the following passages. Lane 1, passage 1; lane 2 , passage 27; lane 3 , passage 47; lane 4 avirulent (ATCC). ( $A$ ) Amido black-stained PVDF membrane. $(B)$ Enhanced chemiluminescence immunoblot of $A$ using 1:500 dilution of adsorbed antiserum. These pictures were produced from OFOTO 2.0 files. peared at the site of tick feeding and did not develop until 412 wk after tick engorgement. Kornblatt and his coworkers (19) attempted to produce EM on the clipped backs of New Zealand White rabbits by $(a)$ feeding presumptively infected $I$. scapularis, $(b)$ injecting infected tick organ homogenates, or $(c)$ injecting a culture of $B$. burgdorferi strain G39/40, passage 50 . Of the 33 rabbits inoculated by these various methods, one of four rabbits fed upon by infected ticks developed an erythematous skin lesion at the site of tick attachment which persisted for $3 \mathrm{~d}$. Silver strains of the lesion biopsies showed spirochetes but cultures were negative. Of the 10 rabbits injected intradermally with tick organ homogenates, one developed an erythematous, indurated lesion at two of the three inoculated sites. These lesions appeared in $2 \mathrm{~d}$ and persisted for 6 . Both silver stains and cultures of these biopsies were negative. None of the 19 rabbits inoculated both intradermally and intravenously with either tick organ homogenates or the G39/40 strain developed EM.

The present study demonstrates that EM lesions can be consistently produced in the rabbit after intradermal inoculation of B. burgdorferi, even after 27 in vitro passages (Table II). Our finding of infiltrating lymphocytes, occasional plasma cells and macrophages within the superficial and deep dermal layers of the EM lesion, with perivascular cuffing, parallels the observations made for the human EM lesion (4). It is significant to note that the B31 strain, passage 47 , was not as consistent in producing EM. The rabbit model of Lyme borreliosis should be useful as a means in addressing whether the EM lesion is spirochete-associated or the result of an exotoxin secreted during spirochetal multiplication, and to define the pathogenesis of early Lyme borreliosis.

With regard to the mechanisms of EM formation, it should be emphasized that the mean time to appearance of EM and its duration were indistinguishable whether $10^{3}$ or $10^{7}$ B. burgdorferi were injected intradermally. In addition, the presence of viable $B$. burgdorferi could be disassociated from the induction 
of EM in cases of partial immunity where EM developed in the 5-7-d period, despite culture negative skin biopsies (see passage 27, Table VI; and HB19, 297, Table VII). Taken together, these observations suggest that the time to appearance of EM correlates better with the kinetics of the rabbit immune response than with the absolute numbers of spirochetes. The rabbit model should also permit the determination of whether vaccination with OspA and other putative protective immunogens confer protection against EM, and whether such protection is linked to resistance against dissemination and persistence in the skin.

Dissemination is a feature of human $(2,4)$ and experimental Lyme borreliosis $(9-18,22)$. In our adult rabbit model, we found that three $B$. burgdorferi strains could be cultured from skin, joint tissue, and spinal cord, one from skin and joint tissue, one from skin and spinal cord, and two only from skin. While preliminary, these findings are in accord with the possibility that $B$. burgdorferi strains differ in regard to their ability to cause visceral infection. Further study is required to ascertain whether certain strains have specific affinity for the central nervous system, and/or joints.

While dissemination is a characteristic of $B$. burgdorferi infection of the rabbit, it is ultimate clearance of the organism from the skin and viscera which distinguishes the rabbit model from other small animal models. Resolution of skin infection accurately predicted resolution of visceral infection for individual rabbits. The fact that infection is resolved by $12 \mathrm{wk}$ in the rabbit model provides a convenient means for studying the ontogeny of the immune mechanisms which clear the infection.

A unique and important feature of the rabbit model of Lyme borreliosis is the demonstration of infection-derived immunity to reinfection in rabbits challenged with homologous strains of B. burgdorferi. In contrast, rodent models do not involve the development of immunity and are characterized by chronic persistence of $B$. burgdorferi infection $(10,12,15-17)$. The extent of the protective immunity induced by untreated infection was directly related to the extent of prior in vitro passage in the case of the B31 strain. Only B31, passage 4 was able to induce complete protection against EM and dermal infection with as few as $4 \times 10^{3}$ spirochetes. In contrast, initial infection with B31 passage 27 led to partial protection against EM together with complete protection against skin infection. B31 strain, passage 47 induced significantly less protection; there was no protection against skin infection, and partial protection against EM.

The above observations imply that certain determinants required for the production of protective immunity are being lost during in vitro passage. Identification of differences between B31 passages 4 and 47 may identify immunogens responsible for induction of complete protective immunity by passage 4 . In studies relating to the identity of these protective immunogens, we found no distinction between the OspA content of passage 4 and passage 47 B31. Further, our studies utilizing ELISA showed no differences in anti-whole $B$. burgdorferi antibody levels between susceptible and immune rabbits. It is likely that potentially protective molecules represent a small fraction of the total protein antigens used in our ELISA analyses and may, therefore, be below the levels of detection and differentiation utilizing these assays.

In order that our findings may be viewed in the proper context, previous studies addressing differences between virulent and avirulent $B$. burgdorferi must be considered. Schwan and coworkers found that the plasmid profile of $B$. burgdorferi strain SH-2-82 changes during in vitro passage and that certain plasmids are lost (24). They also showed that the loss of plasmids correlated with a loss of infectivity for white-footed mice by in vitro passage 15 .

In the present study we have found that $B$. burgdorferi, strain $\mathrm{B} 31$, retains infectivity for 3-wk $\mathrm{C} 3 \mathrm{H} / \mathrm{HeJ}$ mice at least up to in vitro passage 85 . These differences in the preservation of mouse infectivity may relate to methods used for concentrating $B$. burgdorferi. In harvesting and washing cultures for infection of animals, as described in the Methods, the spirochetes were washed three times in heat-inactivated normal rabbit serum diluted 1:1 in PBS. After each centrifugation and resuspension, the spirochetes were allowed to sit at room temperature for $15 \mathrm{~min}$ to regain maximum motility. All spirochetes exhibited normal motility after this harvesting procedure. By comparison Schwan and coworkers reported resuspending the spirochetes in PBS without serum before infection of animals (24), which we feel may reduce motility and virulence of the spirochetes (Foley, D. M., M. A. Lovett, and J. N. Miller, unpublished observations).

In a study addressing compositional differences between virulent B31, passage 4-10, and the avirulent ATCC strain B31, Norris and his coworkers demonstrated that lipoproteins of $28(\mathrm{OspD})$ and $35 \mathrm{kD}$ are found in low passaged $B$. burgdorferi but not in ATCC B31 (31). The gene for OspD is located on a 38-kbp linear plasmid which is present in the low passage strain but not in the ATCC B31 strain.

Our search for antigens related to in vitro passage induction of immunity was based upon the development of an antibody reagent specific for virulent $B$. burgdorferi. This was accomplished by extensive adsorption of serum from a completely immune rabbit with the ATCC B31 strain. Our Western blot analysis of strain B31, passages 1,27, 47, and the ATCC strain confirm and extend the observations of Norris with regard to the association of OspD and a $35-\mathrm{kD}$ protein with virulence of this strain (31). We found that 70-, 55-, 35-, 32-, 28-, 18-, $17.5-$, and $15.5-\mathrm{kD}$ antigens were detected in B31 passage 1 but absent in the ATCC B31 strain. We have confirmed that the $28-\mathrm{kD}$ antigen is the outer membrane lipoprotein OspD which is absent both in passage 47 and in the ATCC B31 strain (Skare, J. T., E. S. Shang, D. M. Foley, D. R. Blanco, C. I. Champion, T. Mirabekov, Y. Sokolov, B. L. Kagan, J. N. Miller, and M. A. Lovett, manuscript in preparation). We have termed protein antigens found in virulent but not ATCC B31, "va", for virulent strain associated. While passage 27 and 47 lacked several va antigens found in passage 1 , it is interesting to note that passage 47 had several va antigens, including $38,33,27$, 17.5 and $17 \mathrm{kD}$, which appeared to be distinct in molecular mass from the va antigens of passages 1 and 27. Further, the $35-\mathrm{kD}$ species observed in passage 47 and the ATCC B31 is not the early passage associated protein described above since this protein is absent in passage 47 as determined by two dimensional electrophoresis (Skare, J. T., E. S. Shang, D. M. Foley, D. R. Blanco, C. I. Champion, T. Mirabekov, Y. Sokolov, B. L. Kagan, J. N. Miller, and M. A. Lovett, manuscript in preparation). It is possible that the patterns of va antigens distinctive of each of the B31 passages represent molecules relevant to the induction of protective immunity.

The homologous challenge experiments with the B31 strain were based upon infection with a given passage but in every case involved subsequent intradermal challenge with B31 passage 4 . We do not know if the in vitro passage related changes we have observed are reversible upon animal infection. To the 
extent that such changes correspond to plasmid loss, we would not expect reversibility. It is possible that while passage 47 infected rabbits might be susceptible to infection with passage 4 , they could be immune to reinfection with passage 47 if, in fact, each passage had a distinctive set of outer membrane proteins. In this regard, studies are planned to determine if passagerelated antigenic changes in strain B31 are reversible, and to learn the cellular location of va antigens. Such information could establish a foundation for the ultimate identification of $B$. burgdorferi molecules involved in both pathogenesis and host immunity.

\section{Acknowledgments}

We thank Colin Barkham, Lupe ChuaTeco, and Norma Perez-Abele for their excellent technical assistance. ELISA statistical analysis was performed by UCLA statistical and biomathematical consulting clinic. This work was completed by Denise M. Foley in partial satisfaction of the requirements for the degree Doctor of Philosophy in Microbiology and Immunology.

Supported by National Institutes of Health (NIH) grant AI-37312 and a gift from Dr. Lin Yeiser Coonan to J. N. Miller, NIH grant AI29733 to M. A. Lovett, and Training Grant 2-T32-AI-07323-06 to D. M. Foley.

\section{References}

1. 1992. Morb. Mortal. Wkly. Rep. 40:1.

2. Steere, A. C. 1989. Lyme disease. N. Engl. J. Med. 321:586-596.

3. Steere, A. C., S. E. Malawista, J. A. Hardin, S. Ruddy, P. W. Askenase, and W. A. Andiman. 1977. Erythema chronicum migrans and Lyme arthritis: the enlarging clinical spectrum. Ann. Intern. Med. 86:685-698.

4. Duray, P. H. 1989. Histopathology of clinical phases of human Lyme disease. Rheum. Dis. Clin. North Am. 15:691-710.

5. Steere, A. C., R. L. Grodzicki, A. N. Kornblatt, J. E. Craft, A. G. Barbour W. Burgdorfer, G. P. Schmid, R. Johnson, and S. E. Malawista. 1983. The spirochetal etiology of Lyme disease. N. Engl. J. Med. 308:733-740.

6. Stanek, G., J. Klein, R. Bittner, and D. Glogar. 1990. Isolation of Borrelia burgdorferi from the myocardium of a patient with longstanding cardiomyopathy N. Engl. J. Med. 322:249-252.

7. Schmidli, J., T. Hunziker, P. Moesli, and U. B. Schaad. 1988. Cultivation of Borrelia burgdorferi from joint fluid 3 months after treatment of facial palsy due to Lyme borreliosis. J. Infect. Dis. 158:905-906.

8. Logigan, E. L., R. F. Kaplan, and A. C. Steere. 1990. Chronic neurologic manifestations of Lyme disease. N. Engl. J. Med. 323:1438-1444.

9. Barthold, S. W., K. D., Moody, G. A. Terwilliger, P. D. Duray, R. O. Jacoby, and A. C. Steere. 1988. Experimental Lyme arthritis in rats infected with Borrelia burgdorferi. J. Infect. Dis. 157:842-846.

10. Moody, K. D., S. W. Barthold, G. A. Terwilliger, D. S. Beck, G. M Hansen, and R. O. Jacoby. 1990. Experimental chronic Lyme borreliosis in Lewis rats. Am. J. Trop. Med. Hyg. 42:165-174.

11. Barthold, S. W., D. S. Beck, G. M. Hansen, G. A. Terwilliger, and K. D.
Moody. 1990. Lyme borreliosis in selected strains and ages of laboratory mice. J. Infect. Dis. 162:133-138.

12. Barthold, S. W., M. S. de Souza, J. L. Janotka, A. L. Smith, and D. H. Persing. 1993. Chronic Lyme borreliosis in the laboratory mouse. Am. J. Pathol. 143:959-971.

13. Schmitz, J. L., R. F. Schell, A. Hejka, D. M. England, and L. Konick. 1988. Induction of Lyme arthritis in LSH hamsters. Infect. Immun. 56:23362342.

14. Johnson, R. C., N. Marek, and C. Kodner. 1984. Infection of Syrian hamsters with Lyme disease spirochetes. J. Clin. Microbiol. 20:1099-1101.

15. Goodman, J. L. P. Jurkovich, C. Kodner, and R. C. Johnson. 1991. Persistent cardiac and urinary tract infections with Borrelia burgdorferi in experimentally infected Syrian hamsters. J. Clin. Microbiol. 29:894-896.

16. Preac-Mursic, V., E. Patsouris, B. Wilske, S. Reinhardt, B. Grob, and P. Mehraein. 1990. Persistence of Borrelia burgdorferi and histopathological alterations in experimentally infected animals: comparison with histopathological findings in human Lyme disease. Infection. 18:332-341.

17. Sonnesyn, S. W., J. C. Manivel, R. C. Johnson, and J. L. Goodman. 1993. A guinea pig model for Lyme Disease. Infect. Immun. 61:4777-4784.

18. Philipp, M. T., M. K. Aydintug, R. P. Bohm, Jr., F. B. Cogswell, V. A. Dennis, H. N. Lanners, R. C., Lowrie, Jr., E. D. Roberts, M. D. Conway, M. Karacorlu, et al. 1993. Early and early disseminated phases of Lyme disease in the rhesus monkey: a model for infection in humans. Infect. Immun. 61:30473059.

19. Kornblatt, A. N., A. C. Steere, and D. G. Brownstein. 1984. Experimental Lyme disease in rabbits: spirochetes found in EM and blood. Infect. Immun. 46:220-223.

20. Burgdorfer, W., A. G. Barbour, S. F. Hayes, J. L. Benach, E. Grunwaldt, and J. P. Davis. 1982. Lyme disease-a tickborne spirochetosis? Science (Wash. DC). 216:1317-1319.

21. Krinsky, W. L., S. J. Brown, and P. W. Askenese. 1982. Ixodes dammini skin lesions in guinea pigs and rabbits compared to erythema chronicum migrans in patients with Lyme arthritis. Exp. Parisitol. 53:381-395.

22. Moody, K. D., S. W. Barthold, and G. A. Terwilliger. 1990. Lyme borreliosis in laboratory animals: effect of host species and in vitro passage of Borrelia burgdorferi. Am. J. Trop. Med. Hyg. 43:87-92.

23. Barbour, A. G. 1984. Isolation and cultivation of Lyme disease spirochetes. Yale J. Biol. Med. 57:521-525.

24. Schwan, T. G., W. Burgdorfer, and C. F. Garon. 1988. Changes in infectivity and plasmid profile of the Lyme disease spirochete, Borrelia burgdorferi as a result of in vitro cultivation. Infect. Immun. 56:1831-1836.

25. Schwan, T. G., W. Burgdorfer, M. E. Schrumpf, and R. H. Karstens. 1988. The urinary bladder: a consistent source of Borrelia burgdorferi in experimentally infected white-footed mice (Peromyscus leocopus). J. Clin. Microbiol. 26:893895.

26. Wilske, B., V. Preac-Mursic, U. B. Gobel, B. Graf, S. Jauris, E. Soutschek E. Schwab, and G. Zumstein. 1993. An OspA serotyping system for Borrelia burgdorferi based on reactivity with monoclonal antibodies and OspA sequence analysis. $J$. Clin. Microbiol. 31:340-350.

27. Laemmli, U. K. 1970. Cleavage of structural proteins during the assembly of the head bacteriophage T4. Nature (Lond.). 227:680-685.

28. Towbin, H., T. Staechelin, and J. Gordon. 1979. Electrophoretic transfer of proteins from polyacrylamide gels to nitrocellulose sheets: procedure and some applications. Proc. Natl. Acad. Sci. USA. 76:4350-4354.

29. Harlow, E., and D. Lane. 1988. Antibodies: A Laboratory Manual. Cold Spring Harbor Laboratory Press, Cold Spring Harbor, N.Y.

30. Fleiss, J. 1986. The Design and Analysis of Clinical Experiments. John Wiley and Sons, N.Y. 46-90.

31. Norris, S. J., C. J. Carter, J. K. Howell, and A. G. Barbour. 1992. Low passage-associated proteins of Borrelia burgdorferi B31: characterization and molecular cloning of OspD, a surface-exposed, plasmid-encoded lipoprotein. Infect. Immun. 60:4662-4672. 